As examples we mention $y^{3}-x^{4}=0, y^{3}-x^{5}=0$, and $\left[y-1+\left(1-x^{2}\right)^{1 / 2}\right]^{2}-x^{5}=0$ with the determination of $\left(1-x^{2}\right)^{1 / 2}$ which equals 1 when $x=0$. These give respectively, at the origin, a minimum, a point of inflection, and a cusp with both branches concave upward. In none of the three cases is $y$ analytic in $x$ at the origin. An example where the locus is a single point is given by $y+i x=0$.

In the case of a reducible function $f(x, y)$, the real locus $f(x, y)=0$ neighboring $\left(x_{0}, y_{0}\right)$ consists of a finite number of configurations of the kind described in the theorem, no two of which have any point except $\left(x_{0}, y_{0}\right)$ in common. This is easily proved by use of theorems on resultants and on divisibility of one function by another. Of course two irreducible factors may have exactly the same locus.

Columbia University

\title{
A PARTIAL DIFFERENTIAL EQUATION CONNECTED WITH THE FUNCTIONS OF THE PARABOLIC CYLINDER*
}

BY HARRY BATEMAN

The partial differential equation

$$
\sum_{s=1}^{p}\left(\frac{\partial^{2} V}{\partial x_{s}^{2}}-x_{s} \frac{\partial V}{\partial x_{s}}\right)+\nu V=0,
$$

which was considered by Mehler $\dagger$ in 1866 , is a slight modification of an equation which occurs in wave-mechanics in the theory of the rotator in a plane and in space. $\$$ The case in which $\nu$ is a positive integer is then of chief physical interest and Mehler's simple solution

$$
V=\prod_{s=1}^{p} H_{m_{s}}\left(x_{s}\right), \quad \sum_{s=1}^{p} m_{s}=\nu,
$$

acquires a physical significance. The function $H_{m}(x)$ is the polynomial of Laplace and Hermite defined by the equation

* Presented to the Society, December 2, 1933.

$\dagger$ F. G. Mehler, Journal für Mathematik, vol. 66 (1866), p. 161.

$\ddagger$ A. Sommerfeld, Atombau und Spektrallinien, wellenmechanischer Ergänzungsband, 1929, p. 23. 


$$
H_{m}(x)=e^{z}\left(-D_{x}\right)^{m} e^{-z}=(2 \pi)^{1 / 2} e^{z} \phi^{(m)}(x),
$$

where $2 z=x^{2}$. The $H$-notation is that adopted by Appell and de Fériet in their book, ${ }^{*}$ the $\phi$-notation is one used in statistics.

When $p=2$ we have the equation

$$
\frac{\partial^{2} V}{\partial x^{2}}+\frac{\partial^{2} V}{\partial y^{2}}-x \frac{\partial V}{\partial x}-y \frac{\partial V}{\partial y}+\nu V=0
$$

which is closely related to one considered by E. L. Ince. $\dagger$ The normal or simple solutions are of type

$$
V=h_{\nu-m}(x) k_{m}(y)
$$

where $h_{s}(x), k_{s}(x)$ are solutions of the differential equation

$$
\frac{d^{2} u}{d x^{2}}-x \frac{d u}{d x}+s u=0 .
$$

Ince generalized this solution by multiplying it by an arbitrary function $f(n)$ and integrating with respect to $n$. We shall use summation instead of integration and shall suppose $m+1$ to be a positive integer. We shall, moreover, put $h_{s}(x)=k_{s}(x)=H_{s}(x)$, where $H_{s}(x)$ is defined for one-half of the $x$ plane and for all values of $s$ by the equation

$$
\begin{aligned}
& C_{m+s, m} m ! i^{m} H_{s}(x) \\
& =\int_{0}^{\infty}\left[(x+i y)^{s+m} \phi^{(m)}(y)+(x-i y)^{s+m} \phi^{(m)}(-y)\right] d y,
\end{aligned}
$$

which reduces to the well known formula of Laplace $\ddagger$ and Mehler§ when $m=0$ and $s$ is a positive integer. On the right-hand side of the equation (7), the many-valued function $(x \pm i y)^{2 n}$ is supposed to be defined for $y>0$ so that when $x \rightarrow 0$ it reduces to $y^{2 n} \exp ( \pm i n \pi)$. When $x$ is complex and unrestricted the integral is not single-valued, but if $x$ is restricted to the half-plane

* P. Appell and J. Kampé de Fériet, Fonctions Hypergéométriques et Hypersphériques, Polynomes d'Hermite, 1926, p. 344.

$\dagger$ E. L. Ince, Proceedings of the Royal Society of Edinburgh, vol. 44 (1924), p. 242.

$\ddagger$ P.S. Laplace, Thêorie Analytiques des Probabilités, Livre 2, Oeuvres, vol. 7, p. 299.

$\S$ F. G. Mehler, loc. cit. 
$R(x)>0$, the integral agrees in value with a single-valued function which satisfies the differential equation (6). It is, indeed, easy to verify by an integration by parts that the definite integral has the form on the left, where $H_{s}(x)$ is some function of $s$ and $x$ such that $H_{s}(x)$ satisfies (6). The proof of the last property depends on the fact that $(x+i y)^{\nu}$ is a solution of the differential equation (4).

To find which solution of (6) the integral represents we first choose $R(s+m)>0$ and make $x \rightarrow 0$. Writing $s=2 r$ and observing that*

(8) $2 \pi \int_{0}^{\infty} y^{m+2 r} \phi^{(m)}(y) d y=C_{2 r+m, m} m ! 2^{r} \Gamma\left(\frac{1}{2}\right) \Gamma\left(r+\frac{1}{2}\right)$,

we find that

$$
\begin{aligned}
H_{2 r}(0) \Gamma\left(\frac{1}{2}-r\right) & =2^{r} \Gamma\left(\frac{1}{2}\right) \\
H_{2 r}^{\prime}(0) \Gamma(-r) & =2^{r-1 / 2} \Gamma\left(-\frac{1}{2}\right) .
\end{aligned}
$$

Hence, with the usual notation for the confluent hypergeometric function (without the suffixes),

$$
\begin{aligned}
H_{2 r}(x) \Gamma(-r)= & 2^{r} B\left(\frac{1}{2},-r\right) F\left(-r ; \frac{1}{2} ; \frac{x^{2}}{2}\right) \\
& +\Gamma\left(-\frac{1}{2}\right) 2^{r-1 / 2} \cdot x F\left(\frac{1}{2}-r ; \frac{3}{2} ; \frac{x^{2}}{2}\right) .
\end{aligned}
$$

In Whittaker's notation, if $s$ is not a negative integer,

$$
H_{s}(x)=\exp \left(\frac{x^{2}}{4}\right) D_{s}(x)
$$

This equation may be used to define the function $H_{s}(x)$ for all real and complex values of $x$. An equation equivalent to this has

* The value of the integral is given in a slightly different form by G. N. Watson, Proceedings of the London Mathematical Society, vol. 8 (1910), p. 393. 
indeed been used for this purpose* for all real and complex values of $s$ except negative integral values. When $R(s+m)>0$ we can, by integrating the integral in (7) a sufficient number of times, pass to the case in which $R(s+m)<0$. An exceptional case arises when $s$ is a negative integer. If we take $m+s$ to be a negative integer, the integral has a definite value and gives a definition of $H_{s}(x)$, but a different notation is used for the function thus defined. $\dagger$ If $m+s$ is a positive integer, the integral and $C_{m+s, m}$ are both zero for $s<0$. When $s$ is a positive integer $p-n$, the relation ( 7$)$ gives at once the expansion

$$
(x+i y)^{p}=\sum_{m=0}^{p} C_{p, m} i^{m} H_{p-m}(x) H_{m}(y),
$$

which may be checked by comparing the terms of degree $p$ on the two sides of the equation and noting that both sides satisfy the partial differential equation (4). This equation may be used to derive from (7) the expansion ( $s$ not a negative integer)

$$
H_{s+p}(x)=\sum_{m=0}^{p}(-1)^{m} m ! C_{p, m} C_{s, m} H_{s-m}(x) H_{p-m}(x),
$$

which may be inverted so as to give an expansion for the product in the form $\ddagger$

$$
H_{s}(x) H_{n}(x)=\sum_{m=0}^{n} m ! C_{s, m} C_{n, m} H_{n+s-2 m}(x) .
$$

This relation may be established directly by induction. $\S$ When $s=n$ the equation takes the form

$$
\left[H_{n}(x)\right]^{2}=\sum_{s=0}^{n} C_{n, s} n !(s !)^{-1} H_{2 s}(x)
$$

* M. Plancherel and W. Rotach, Commentarii Mathematici Helvetici, vol. 1 (1929), p. 227. See also S. C. van Veen, Amsterdam Proceedings, vol. 34 (1931), p. 257.

† G. N. Watson, loc. cit.; O. Volk, Mathematische Annalen, vol. 86 (1922), p. 296; Appell and de Fériet, loc. cit., p. 362.

$\ddagger$ An equivalent expansion is given by Gorakh Prasad for positive integral values of $s$ and $n$. Products of Hermite polynomials are considered also by N. Nielsen, Recherches sur les Polynomes de Hermite, Copenhagen, 1918.

$\S$ Gorakh Prasad, Proceedings of the Benares Society, vol. 2 (1920), p. 18. 
This series may be compared with Mitra's series* for $D_{n}^{2}(x)$,

(16) $\left[D_{n}(x)\right]^{2}=\sum_{s=0}^{\infty}(2 s-1) ! !(2 n-2 s-1) ! !\left[(2 s) ! ! 2^{n+s+1 / 2}\right]^{-1} H_{2 s}(x)$,

which has been written in an abbreviated form with the aid of Schuster's notation $\dagger$

$$
\begin{aligned}
(2 s) ! ! & =(2 s)(2 s-2) \cdots(2), \\
(2 s+1) ! ! & =(2 s+1)(2 s-1) \cdots(1),
\end{aligned}
$$

and a natural extension of it, namely,

$$
(-1-2 s) ! !(-1)(-3) \cdots(1-2 s)=1 \text {. }
$$

Mitra's expansion may be generalized with the aid of the well known recurrence relations

$$
\begin{gathered}
D_{n+1}(x)-x D_{n}(x)+n D_{n-1}(x)=0 \\
D_{n}^{\prime}(x)+\frac{1}{2} x D_{n}(x)-n D_{n-1}(x)=0 \\
H_{n+1}(x)-x H_{n}(x)+n H_{n-1}(x)=0 \\
H_{n}^{\prime}(x)=n H_{n-1}(x) .
\end{gathered}
$$

The resulting equations are

$$
\begin{aligned}
& D_{n}(x) D_{n-2 r}(x) \\
& =\sum_{s=0}^{\infty}(2 r+2 s-1) ! !(2 n-2 r-2 s-1) ! !(2 s-2 r-1) ! ! A_{s}
\end{aligned}
$$

where

$$
\begin{aligned}
& A_{s} \cdot(2 s) ! !(2 s-1) ! ! 2^{n-r+s+1 / 2}=H_{2 s}(x) \\
& D_{n}(x) D_{n-2 r+1}(x) \\
& =\sum_{s=0}^{\infty}(2 r+2 s-1) ! !(2 n-2 r-2 s-1) ! !(2 s-2 r+1) ! ! C_{s}
\end{aligned}
$$

where 27.

* S. C. Mitra, Proceedings of the Edinburgh Society, (2), vol. 4 (1934), p.

$\dagger$ A. Schuster, Philosophical Transactions, Royal Society of London, (A), vol. 200 (1903), p. 181. The extension is also given by Schuster. 


$$
C_{s}(2 s) ! !(2 s+1) ! ! 2^{n-r+s+3 / 2}=H_{2 s+1}(x) .
$$

These equations may be checked* by comparing them with (13) and (14) after these have been multiplied throughout by $D_{0}^{2}(x)$ so that they become equations between $D$-functions. The verification depends on the identities

$$
\begin{aligned}
& (2 m+2 n-1) ! !(-2 n-1) ! !(2 r-1) ! ! \\
& =\sum_{s=0}^{m} 2^{s} C_{m, s} C_{m+n+r, s} s !(2 m+2 r-2 s-1) ! ! \\
& \cdot(2 s-2 m-2 n-1) ! !(2 m+2 n-2 s-1) ! ! \\
& (2 n+2 s-1) ! !(2 n-2 s-1) ! !(2 s-2 n-1) ! ! \\
& =\sum_{m=0}^{p}(-2)^{m} C_{p, m} C_{2 n-p, m} m !(2 p-2 n+2 s-1) ! ! \\
& \cdot(2 n-2 m-2 s-1) ! !(2 s+2 n-2 p-1) ! !
\end{aligned}
$$

Let us now consider the integral

$$
H_{\nu, m}(\alpha, x)=\int_{-\infty}^{\infty} H_{\nu}(x \cos \alpha+y \sin \alpha) \phi^{(m)}(y) d y,
$$

which is convergent when $-\pi<2 \alpha<\pi$ if $H_{\nu}(z)$ is defined by (11), for the behavior of $D_{\nu}(z)$ when $z$ is large is known. Since

$$
\frac{\partial}{\partial z} H_{\nu}(z)=\nu H_{\nu-1}(z)
$$

we readily find that

$$
\begin{aligned}
\frac{\partial}{\partial \alpha} H_{\nu, 0}(\alpha, x)= & -x \sin \alpha \int_{-\infty}^{\infty} \nu H_{\nu-1}() \phi^{(0)}(y) d y \\
& +\cos \alpha \int_{-\infty}^{\infty} \nu H_{\nu-1}() y \phi^{(0)}(y) d y \\
= & -\nu x \sin \alpha H_{\nu-1,0}(\alpha, x) \\
& +\nu(\nu-1) \cos \alpha \sin \alpha H_{\nu-2,0}(\alpha, x) .
\end{aligned}
$$

* The expansions obtained by putting $n=2 r$ in (21) and $n=2 r-1$ in (22) have already been given by R. K. Varma in a slightly different form, Proceedings of the Benares Society, vol. 10 (1928), p. 11. Some special cases were considered previously by Gorakh Prasad, Proceedings of the Benares Society, vol. 2 (1920), p. 12. 
Since, when $\alpha \rightarrow 0$,

$$
H_{\nu, 0}(0, x)=H_{\nu}(x)=x H_{\nu-1}(x)-(\nu-1) H_{\nu-2}(x),
$$

we find that a solution of equation (25) is

$$
H_{\nu, 0}(\alpha, x)=\cos ^{\nu} \alpha H_{\nu}(x) .
$$

Integrating the integral (24) by parts $m$ times we find

$$
H_{\nu, m}(\alpha, x)=C_{\nu, m} m ! \cos ^{\nu-m} \alpha \sin ^{m} \alpha H_{\nu-m}(x) .
$$

The associated equation*

$$
\begin{aligned}
& H_{\nu}(x \cos \alpha+y \sin \alpha) \\
& =\sum_{m=0}^{\infty} C_{\nu, m} \cos ^{\nu-m} \alpha \sin ^{m} \alpha H_{\nu-m}(x) H_{m}(y)
\end{aligned}
$$

was obtained by Kampé de Fériet $\dagger$ in the case when $\nu$ is a positive integer by using the generating function of the Hermite polynomial, a method which he used also to obtain an expansion for $H\left(p_{1} x_{1}+p_{2} x_{2}+\cdots+p_{s} x_{s}\right)$ in a series of simple functions of type (1) on the supposition that $p_{1}^{2}+p_{2}^{2}+\cdots=1$. In the general case when $\nu$ is not a positive integer we may consider the series in powers of $\tan \alpha$ of $\sec ^{\nu} \alpha H_{\nu}(x \cos \alpha+y \sin \alpha)$. If $\nu$ is not a negative integer, the radius of convergence of this series is determined by the location of the singularities of $\sec ^{\nu} \alpha$, for $H_{\nu}(z)$ is uniform and analytic over the whole of the finite part of the $z$-plane. The power series for $\sec ^{\nu} \alpha H_{\nu}($ ) is thus convergent for $-\pi<2 \alpha<\pi$, and so to establish the truth of the series under this condition it is only necessary to prove that if $t=\tan \alpha$, then

$$
\begin{aligned}
I(m, \nu) & =\left[\frac{d^{m}}{d t^{m}} \sec ^{\nu} \alpha H_{\nu}(x \cos \alpha+y \sin \alpha)\right]_{t=0} \\
& =C_{\nu, m} m ! H_{\nu-m}(x) H_{m}(y) .
\end{aligned}
$$

* When $\nu$ is a positive integer this equation may be checked by observing that both sides are polynomial solutions of (4) with the same terms of degree $\nu$ and these terms determine the solution uniquely.

$\dagger J$. Kampé de Fériet, Danske Videnskabernes Selskabs, MathematiskFysiske Meddelelser, vol. 5 (1923), No. 2. See also Appell and de Fériet, loc. cit., and V L. Mutatker, Journal of the Indian Mathematical Society, vol. 1 (1934), p. 53. The case in which $\alpha=\pi / 4$ is due to C. Runge, Mathematische Annalen, vol. 75 (1914), p. 130. The equation was in fact, used by him to solve a special type of non-linear integral equation. 
This proof may be carried out by induction. Thus, if we suppose that $\sec ^{\nu} \alpha H_{\nu}(x \cos \alpha+y \sin \alpha)=F(\nu, ' t)$, we have, on differentiating first once and then $m$ times,

$$
\begin{aligned}
\left(1+t^{2}\right) \frac{d}{d t} F(\nu, t)= & (y-x t) \nu F(\nu-1, t)+\nu t F(\nu, t), \\
I(m+1, \nu)= & y \nu I(m, \nu-1)-m x \nu I(m-1, \nu-1) \\
& +m(\nu-m-1) I(m-1, \nu),
\end{aligned}
$$

for $t=0$, and the recurrence relation is satisfied by the expression on the extreme right of (30).

When $\nu$ is a positive integer, the integral representing $H_{\nu, 0}(\alpha, x)$ may be evaluated by putting $z=x \cos \alpha+y \sin \alpha$ and using Mehler's expansion*

$$
\begin{gathered}
\csc \alpha \cdot \exp \left[\csc ^{2} \alpha\left(x^{2} \cos ^{2} \alpha-2 x z \cos \alpha+z^{2} \cos ^{2} \alpha\right)\right] \\
=\sum_{s=0}^{\infty} \frac{1}{s !} \cos ^{8} \alpha \cdot H_{s}(x) H_{s}(z) .
\end{gathered}
$$

When the integral for $H_{\nu, m}(\alpha, x)$ is treated in the same way, we obtain the integral

$$
\begin{aligned}
& \int_{-\infty}^{\infty} H_{\nu}(z) \phi_{m}(z \csc \alpha-x \operatorname{ctn} \alpha) d z \\
& =C_{\nu, m} m ! \cos ^{\nu-m} \alpha \sin ^{m} \alpha H_{\nu-m}(x),
\end{aligned}
$$

which gives the expansion

$$
\begin{aligned}
& e^{z^{2} / 2} \phi_{m}(z \csc \alpha-x \operatorname{ctn} \alpha) \\
& \quad=(2 \pi)^{-1 / 2} \sum_{n=m}^{\infty}[(n-m) !]^{-1} \cos ^{n-m} \alpha \sin ^{m} \alpha H_{n}(z) H_{n-m}(x) .
\end{aligned}
$$

Another expansion may be obtained by writing $\operatorname{ctn} \alpha=e^{-t}$ and observing that the function $V=\csc ^{\nu} \alpha \cdot H_{\nu}(x \cos \alpha+y \sin \alpha)$ is a solution of the partial differential equation $\dagger$

* F. G. Mehler, loc. cit.

$\dagger$ This equation may be transformed into one which Laplace solved by means of a definite integral, Théorie Analytique des Probabilités, Livre 2, Oeuvres, vol. 7, p. 294. The equation was used later by Smoluchowski in his theory of the Brownian movement. See B. Hostinsky, Annales de l'Institut Poincaré, vol. 3 (1932), pp. 1-72. 


$$
\frac{\partial^{2} V}{\partial x^{2}}-x \frac{\partial V}{\partial x}=\frac{\partial V}{\partial t}
$$

If this equation has a solution of the form

$$
V=\sum_{k=0}^{\infty} C_{k}(t) x^{k} / k !,
$$

the coefficient $C_{k}(t)$ must satisfy the differential-difference equation

$$
C_{k}^{\prime}(t)+k C_{k}(t)=C_{k+2}(t),
$$

and if the value of $C_{k}(0)$ is known, an appropriate solution is

$$
C_{k}(t)=\sum_{s=0}^{\infty} 2^{-s} C_{k+2 s}(0) e^{-k t}\left(1-e^{-2 t}\right)^{s} / s !
$$

Instead of defining $C_{k}(t)$ as a coefficient we may define it by the equation

$$
C_{k}(t)=\left(\frac{\partial^{k} V}{\partial x^{k}}\right)_{x=0} .
$$

In particular, when $V=\csc ^{\nu} \alpha \cdot H_{\nu}(x \cos \alpha+y \sin \alpha)$, we have

$$
C_{k}(t)=C_{\nu, k} k ! \cos ^{k} \alpha \csc ^{\nu} \alpha H_{\nu-k}(y \sin \alpha),
$$

and if $n=\nu-k$, the foregoing expansion for $C_{k}(t)$ leads to the equation

$$
\begin{aligned}
& H_{n}(y \sin \alpha) \\
& =\sum_{s=0}^{\infty} C_{n, s} H_{n-s}\left(y(2)^{1 / 2}\right) H_{s}(0) 2^{(n-s) / 2} \sin ^{n-s} \alpha \cos ^{s} \alpha,
\end{aligned}
$$

which may be rewritten in the form

$$
H_{n}(u v)=\sum_{s=0}^{\infty} C_{n, s} H_{n-s}(u) H_{s}(0) v^{n-s}\left(1-v^{2}\right)^{s / 2} .
$$

When $n$ is a positive integer, this expansion is equivalent to one given by Appell and de Fériet.* The associated integral

* Appell and de Fériet, loc. cit., p. 346. Equation (40) is related also to equation (67) in the paper of S. Goldstein, Proceedings of the London Mathematical Society, (2), vol. 34 (1932), p. 103. 


$$
\int_{-\infty}^{\infty} H_{m}(u v) \phi_{m-s}(u) d u=m ! H_{s}(0) v^{m-s}\left(1-v^{2}\right)^{s / 2} / s !
$$

is closely connected with one given by Doetsch.*

If in the expansion (29) we put $\alpha=\pi / 4$ and set first $x=u(2)^{1 / 2}$, $y=v(2)^{1 / 2}$, secondly $x=(u+v)(2)^{1 / 2}, y=0$, we obtain two expansions for $H_{\nu}(u+v)$. Equating them and writing $x$ for $u(2)^{1 / 2}$, $y$ for $v(2)^{1 / 2}$, we obtain the equation (for $\nu>0$ )

$$
\sum_{m=0}^{\infty} C_{\nu, m} H_{\nu-m}(x) H_{m}(y)=\sum_{m=0}^{\infty} C_{\nu, m} H_{\nu-m}(x+y) H_{m}(0) .
$$

This was obtained by Varma for the case in which $\nu$ is a positive integer. When $x=y$ Runge's expansion

$$
2^{\nu} H_{\nu}\left(x(2)^{1 / 2}\right)=\sum_{m=0}^{\infty} C_{\nu, m} H_{\nu-m}(x) H_{m}(x)
$$

suggests that it may be profitable to study the expansion of a product $H_{r}(x) H_{s}(x)$ in a series of functions of type $H_{n}\left(x(2)^{1 / 2}\right)$. Such expansions have been studied by Mitra. $\ddagger$

California Institute of Technology

* G. Doetsch, Mathematische Zeitschrift, vol. 32 (1930), p. 587.

$\dagger$ R. S. Varma, Proceedings of the Benares Society, vol. 9 (1927), p. 31.

$\ddagger$ S. C. Mitra, Bulletin of the Calcutta Mathematical Society, June, 1926;

Proceedings of the Benares Mathematical Society, vol. 9 (1927), p. 21; Proceedings of the Edinburgh Mathematical Society, (2), vol. 4 (1934), p. 27. See also S. C. Dhar, Journal of the Indian Mathematical Society, new ser., vol. 1 (1934), p. 105. 